07,14

\title{
Специфика статистики упругих и деформационно-прочностных свойств высокопрочных волокон полипропилена
}

\author{
() Ю.М. Бойко ${ }^{1}$, В.А. Марихин ${ }^{1}$, О.А. Москалюк ${ }^{2}$ Л.П. Мясникова ${ }^{1}$ \\ ${ }^{1}$ Физико-технический институт им. А.Ф. Иофрфе РАН, \\ Санкт-Петербург, Россия \\ ${ }^{2}$ Санкт-Петербургский государственный университет технологии и дизайна, \\ Санкт-Петербург, Россия \\ E-mail: yuri.boiko@mail.ioffe.ru
}

Поступила в Редакцию 7 сентября 2021 г.

В окончательной редакции 7 сентября 2021 г.

Принята к публикации 15 сентября 2021 г.

Закономерности статистических распределений комплекса механических характеристик, включая модуль упругости $(E)$, прочность $(\sigma)$ и деформацию при разрыве $\left(\varepsilon_{b}\right)$, высокопрочных промышленных ориентированных волокон полипропилена (ПП) проанализированы с помощью моделей Вейбулла и Гаусса на базе большого массива измерений (по 50 идентичных образцов в каждой серии). Рассчитаны значения статистического модуля Вейбулла $(m)$ - параметра, характеризующего разброс измеренных значений массивов данных $E, \sigma$ и $\varepsilon_{b}$ для образцов ПП двух типов: единичных волокон (мононитей) и полифиламентных волокон, состоящих из нескольких сотен единичных волокон. Для полифиламентных волокон ПП получено более корректное описание распределений $E, \sigma$ и $\varepsilon_{b}$ как в рамках нормального распределения (распределения Гаусса), так и в рамках распределения Вейбулла по сравнению с описанием таких распределений для мононитей ПП. Анализируется влияние конформации цепи полимера на закономерности статистических распределений $E$, $\sigma$ и $\varepsilon_{b}$ для высокопрочных ориентированных полимерных материалов с различным химическим строением цепи и на корректность их описания в рамках моделей Гаусса и Вейбулла. С этой целью сопоставляются значения $m$, рассчитанные в настоящей работе для ПП, имеющего спиралевидную конформацию цепи, со значениями $m$, определенными нами ранее для сверхвысокомолекулярного полиэтилена и полиамида-6 с конформациями цепей в виде плоского транс-зигзага.

Ключевые слова: полипропилен, механические свойства, статистический анализ, распределение Вейбулла, распределение Гаусса.

DOI: $10.21883 /$ FTT.2022.01.51836.199

\section{1. Введение}

Высокопрочные высокомодульные полимерные материалы являются перспективными армирующими материалами для применения в различных актуальных областях техники, требующих повышенных механических характеристик (бронезащита, ракетная техника, авиа- и судостроение и т.д.), поскольку для них удается достигнуть высоких значений прочности $(\sigma=2-6 \mathrm{GPa})[1-5]$ и модуля упругости $(E=230 \mathrm{GPa})[6]$, сопоставимых с характеристиками неорганических материалов [7-16]. Отличительной особенностью некоторых полимерных материалов (как правило, высокоориентированных) является то, что удельные характеристики их механических свойств, нормированные по плотности материала $(\rho)$, являются рекордными по сравнению со всеми другими материалами. Например, для высокопрочной стали с достигнутыми в настоящее время наибольшими значениями $\sigma=1-2 \mathrm{GPa}$ [14-16] при плотности $\rho \approx 8000 \mathrm{~kg} / \mathrm{m}^{3}$ отношение $\sigma$ к $\rho$ составляет $\sigma / \rho=(0.13-0.25) \cdot 10^{-3} \mathrm{GPa} \cdot \mathrm{m}^{3} / \mathrm{kg}$ в то время как для ультраориентированных гель-волокон сверхвысокомолекулярного полиэтилена (СВМПЭ) с $\sigma=6 \mathrm{GPa}$ [1-3] и $\rho \approx 1000 \mathrm{~kg} / \mathrm{m}^{3}$ величина $\sigma / \rho$ может достигать $6 \cdot 10^{-3} \mathrm{GPa} \cdot \mathrm{m}^{3} / \mathrm{kg}$, т.е. быть в $\sim 25-50$ раз больше, чем для стали. Величина этой удельной характеристики для СВМПЭ может уменьшаться по сравнению с другими типами высокопрочных материалов неорганической природы, например, кварцевыми волокнами с $\sigma=6 \mathrm{GPa}$ [9]. И все-таки она остается весьма заметной даже в этом случае, так как значения $\sigma / \rho$ для кварцевых волокон при $\rho \approx 2600 \mathrm{~kg} / \mathrm{m}^{3}$ составит $\sigma / \rho=2.3 \cdot 10^{-3} \mathrm{GPa} \cdot \mathrm{m}^{3} / \mathrm{kg}$, что все еще в $\sim 3$ раза меньше по сравнению со значением $\sigma / \rho$ для СВМПЭ.

Однако неизбежным следствием достижения рекордно высоких значений прочности полимерных материалов (до $6 \mathrm{GPa}$ ) является повышение их жесткости и, как следствие, возникновение при малейших изгибах и сжатиях поверхностных структурных дефектов ротационного типа - так называемых полос сброса, генерирующих появление локализованных микро- и макротрещин [1-3]. Показано [3], что вследствие этих эффектов резко увеличивается разброс экспериментальных значений прочности, обнаруживаемый при испытаниях большой серии идентичных образцов. В таких случаях с целью определения статистически достоверного усред- 
ненного значения $\sigma\left(\sigma_{\mathrm{av}}\right)$ необходимо получение большого массива данных (несколько десятков параллельных измерений) [3,7-13] вместо обычно применяемых на практике испытаний не более пяти образцов [17-22]. Подобный подход позволяет не только надежно определить величину $\sigma_{\mathrm{av}}$, но и установить тип статистического распределения $\sigma$, что представляется очень важным для более глубокого понимания механизмов разрушения материалов.

В ряде работ показано (см., например, [3,5,7-13]), что наиболее корректно распределение прочности хрупких и квазихрупких сверхпрочных материалов описывается статистикой Вейбулла, изначально предложенной именно для этого класса материалов [23]. В то же время, модель Гаусса, наиболее часто используемая для описания статистики широкого ряда свойств, явлений и процессов различной природы, может оказаться не корректной при описании статистики механических свойств сверхпрочных квазихрупких материалов. По нашему мнению, причина пригодности той или иной статистической модели основывается на различии подходов, лежащих в основе представлений о механизмах разрушения высокопрочных ориентированных и обычных изотропных материалов. Действительно, если распределение Гаусса (часто называемое нормальным распределением) (см., например, $[24,25])$ предполагает равновероятностный характер разрушения во всем объёме образца, то в модели Вейбулла [3,5,7-13] в качестве ключевого фактора рассматривается наличие опасных дефектов - микро- и макроскопических трещин, локализованных на поверхности образца. Кроме того, как было показано в наших предыдущих работах [3,26-30], корректность применимости той или иной статистической модели определяется деформационным ресурсом материала, т.е. соотношением хрупкости/пластичности материала. Более того, в некоторых случаях (для более пластичных материалов) нами было продемонстрировано проявление „статистического дуализма“, заключающегося в возможности описания одного и того же массива экспериментальных значений прочности при использовании как модели Гаусса, так и модели Вейбулла [27-29]. Необходимо также отметить, что критичным в этом смысле оказался и тип образца высокопрочного ориентированного полимерного материала, в частности, представляет ли собой образец единичное моноволокно с достаточно большим (для волокон) диаметром сечения $(\sim 100-200 \mu \mathrm{m})$ или совокупность нескольких сотен гораздо более тонких единичных волокон (диаметром $\sim 1 \mu \mathrm{m})$ в виде пучка, который сам по себе представляет статистический объект. По нашему мнению, данное обстоятельство является благоприятным фактором для возможности корректной применимости статистики Гаусса для полифиламентного образца, даже если образец является квазихрупким.

Необходимо также отметить, что применимость модели Вейбулла не ограничивается исключительно описанием распределения прочности, а может быть распростра- нена и на описание статистики других не менее важных механических характеристик - модуля упругости и деформации при разрыве $\left(\varepsilon_{b}\right)[27,29]$. Следовательно, появляется возможность более полной характеризации высокопрочных ориентированных полимерных материалов за счет расширения числа анализируемых статистических механических свойств.

В серии наших предыдущих работ [3,26-30] было проведено детальное исследование статистических закономерностей механического поведения высокопрочных ориентированных полимерных материалов на основе линейных полимеров, СВМПЭ и полиамида-6 (ПА-6), с конформацией цепи (взаимным расположением участков цепи в пространстве) в виде плоского транс-зигзага [31]. Однако, насколько нам известно, высокопрочные материалы на основе полимеров с существенно отличающимся типом конформации цепи не исследовались в данном контексте. Одним из таких полимеров является полипропилен (ПП) со спиралевидной конформацией цепи [31]. Можно ожидать, что изменение типа конформации цепи может оказать существенное влияние на характер статистического распределения механических свойств материалов на основе ПП.

Таким образом, целью работы является анализ статистических распределений комплекса упругих и деформационно-прочностных свойств ориентированных единичных и полифиламентных волокон полипропилена при использовании статистических моделей Гаусса и Вейбулла.

Следует отметить, что модель Гаусса является наиболее распространенной при статистическом анализе баз данных различной природы, в то время как модель Вейбулла гораздо реже используется для этих целей. Поэтому рассмотрим основные положения модели Вейбулла более подробно.

\section{2. Статистика Вейбулла}

В статистической модели Вейбулла вероятность разрушения идентичных образцов при данном или более низком значении приложенного механического напряжения $\sigma$ определяется как

$$
P(\sigma)=1-\exp \left[-\left(\sigma / \sigma_{0}\right)^{m}\right],
$$

где $m-$ так называемый модуль Вейбулла, статистический параметр, являющийся мерой дисперсии результатов измерений, $\sigma_{0}-$ масштабный параметр, имеющий физический смысл среднего значения прочности $\left(\sigma_{\mathrm{av}}\right)[7-13,32]$. Для проведения анализа полученный массив результатов испытаний для $n$ образцов располагается в порядке возрастания величины $\sigma$ и преобразуется в экспериментальное распределение вероятностей с присвоением $j$-му результату совокупной вероятности разрушения $P_{j}$. Величина $P_{j}$ определяется с помощью довольно простых соотношений $P_{j}=f(j, n)$, наиболее 
корректным из которых оказалось уравнение (2) [32]:

$$
P_{j}=(j-0.5) / n \text {. }
$$

После двойного логарифмирования левой и правой частей уравнения (1) и замены $P(\sigma)$ на $P_{j}$ получаем уравнение (3):

$$
\ln \ln \left[1 /\left(1-P_{j}\right)\right]=-m \cdot \ln \sigma_{0}+m \cdot \ln \sigma .
$$

Уравнение (3) представляет собой достаточно простое линейное уравнение

$$
y=a+b x,
$$

где $\quad y=\ln \ln \left[1 /\left(1-P_{j}\right)\right], \quad a=-m \ln \sigma_{0}, \quad b=m \quad$ и $x=\ln \sigma$. Определив $m$ как угол наклона зависимости $\ln \ln \left[1 /\left(1-P_{j}\right)\right]=f(\ln \sigma) \quad$ с $\quad$ помощью $\quad$ стандартной процедуры линейного регрессионного анализа, можно определить параметр $\sigma_{0}$ путем решения уравнений (5) и (6):

$$
\begin{gathered}
\ln \sigma_{0}=-a / m, \\
\sigma_{0}=\exp (-a / m) .
\end{gathered}
$$

Аналогичным образом может быть проведен анализ распределений $\varepsilon_{b}$ и $E[27,29]$ :

$$
\begin{gathered}
\ln \ln \left[1 /\left(1-P_{j}\right)\right]=-m \cdot \ln \varepsilon_{0}+m \cdot \ln \varepsilon_{b}, \\
\varepsilon_{0}=\exp (-a / m), \\
\ln \ln \left[1 /\left(1-P_{j}\right)\right]=-m \cdot \ln E_{0}+m \cdot \ln E, \\
E_{0}=\exp (-a / m) .
\end{gathered}
$$

Уравнения (7) и (9), где $x=\ln \varepsilon_{b}$ или $\ln E$, и $a=-m \cdot \ln \varepsilon_{0}$ или $-m \cdot \ln E_{0}$, также представляют собой простое линейное уравнение (4), что делает их привлекательными для анализа больших массивов измерений $\varepsilon_{b}$ и $E$.

\section{3. Экспериментальная часть}

\section{1. Образцы}

Образцы для исследования представляли собой высокопрочные ориентированные промышленные моноволокна с диаметром образца $0.17 \mathrm{~mm}$ и полифиламентные волокна ПП с линейной плотностью 1600 tex (соответствует эффективному диаметру образца $0.05 \mathrm{~mm}$ ) отечественного производства.

\section{2. Механические испытания}

Для определения разрывной прочности образцы монои полифиламентных волокон длиной $50 \mathrm{~cm}$ подвергались растяжению на разрывной машине Instron-1122 при комнатной температуре при скорости растяжения $200 \mathrm{~mm} / \mathrm{min}$ в соответствии с ГОСТ 6611.2-73, т.е. при скорости деформирования $0.4 \mathrm{~min}^{-1}$. Для исследованных образцов использовались специальные цилиндрические зажимы от фирмы производителя, предусматривающие закрепление и намотку нескольких витков волокна на поверхность цилиндров с целью предотвращения выскальзывания образца из зажимов. Для получения статистически достоверных результатов было протестировано по 50 идентичных образцов моно- и полифиламентов ПП, т. е. суммарно 100 образцов.

\section{4. Результаты и их обсуждение}

На рис. 1 приведены типичные кривые растяжения для образцов моно- и полифиламентов ПП. Видно, что величина деформации при разрыве $\varepsilon_{b}$ составляет около $10 \%$ для образцов двух типов, что в первом приближении позволяет отнести их к квазихрупким материалам, для которых можно ожидать выполнения уравнения (3) для прочности в рамках модели Вейбулла. С этой целью результаты измерений прочности были расположены в порядке возрастания их значений в зависимости от номера образца $n$ (см. рис. $2, a)$ и затем перестроены в координатах $\ln \ln \left[1 /\left(1-P_{j}\right)\right]=f(\ln \sigma)$ (см. рис. $2, b$ ). Результаты анализа полученных данных приведены в табл. 1. Линейные аппроксимации каждой из представленных на рис. 2 зависимостей при компьютерной обработке при использовании одной прямолинейной аппроксимирующей линии (стандартная функция распределения Вейбулла) были получены с достаточно высокими значениями коэффициента детерминации $R^{2}>0.95$. Следовательно, результаты проведенной аппроксимации представляются корректными. При этом можно отметить, что аппроксимация для полифиламента $\left(R^{2}=0.973\right)$ представляется более корректной по сравнению с монофиламентом $\left(R^{2}=0.954\right)$. Кроме того, необходимо также отметить двукратное увеличение величины $m$ для полифиламента $(m=23.02)$ по сравнению

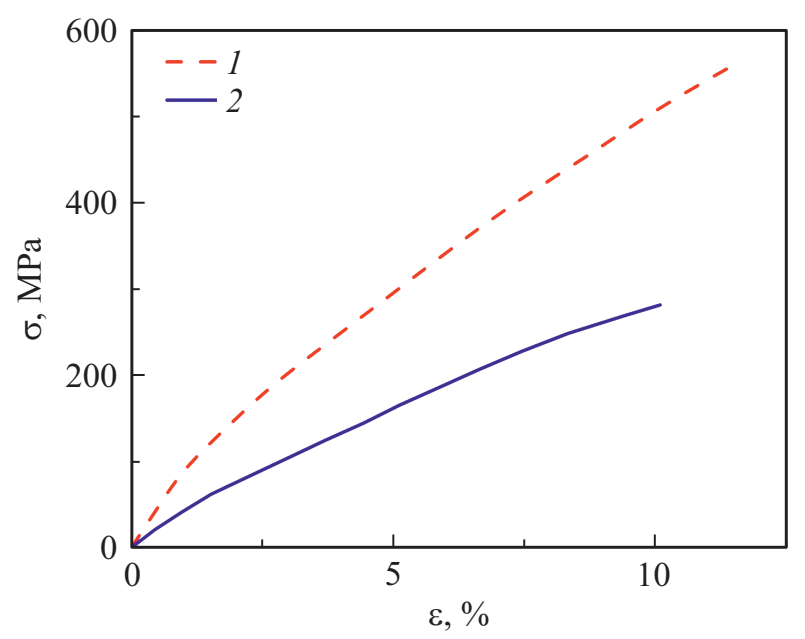

Рис. 1. Кривые растяжения для высокопрочных ориентированных моно- (1) и полифиламентных волокон ПП (2). 

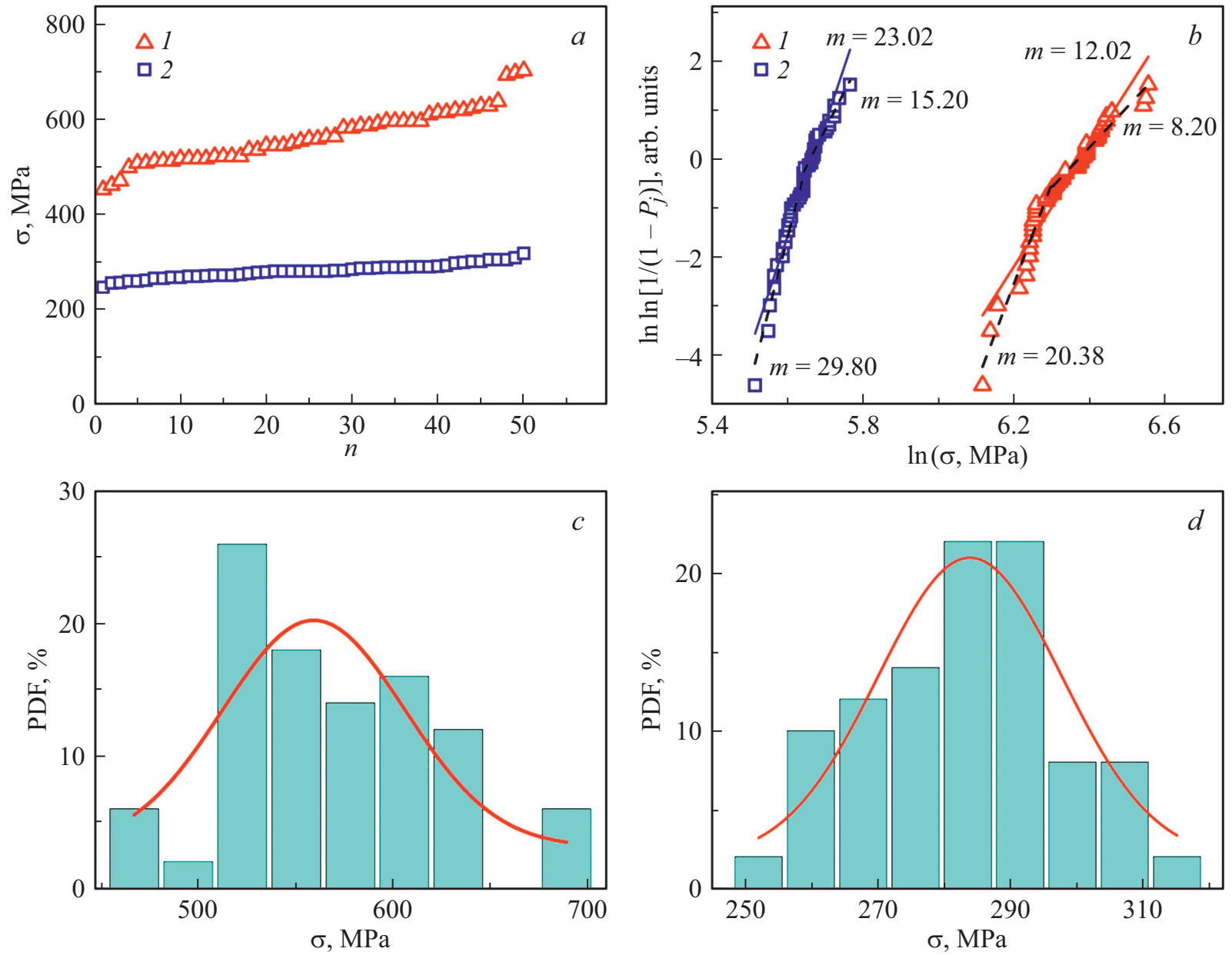

Рис. 2. $a$ - прочность при растяжении $\sigma$ в порядке возрастания ее величины в зависимости от номера образца $n$ для единичных (1) и полифиламентных волокон ориентированного ПП (2); $b$ - графики Вейбулла, построенные из данных, представленных в $(a)$; сплошными и пунктирными линиями показаны результаты линейной аппроксимации при использовании прямой с одним наклоном и двух прямых с различными наклонами, соответственно; $c, d-$ гистограммы функции плотности вероятности $(\mathrm{PDF})$ для прочности единичного $(c)$ и полифиламентного волокна ПП $(d)$ и их описание при использовании функции Гаусса (сплошные колоколообразные кривые).

Таблица 1. Результаты анализа распределения прочности высокопрочных моно- и полифиламентных волокон полипропилена в рамках модели Вейбулла

\begin{tabular}{c|l|c|r|r|r|c}
\hline Тип образца & $y=a+b x$ & $R^{2}$ & $m$ & $\sigma_{0}, \mathrm{MPa}$ & $\sigma_{\mathrm{av}}, \mathrm{MPa}$ & $\sigma_{0} / \sigma_{\mathrm{av}}$ \\
\hline Монофиламент & $y=-76.68+12.02 x$ & 0.954 & 12.02 & 590 & 570 & 1.04 \\
& $y=-128.89+20.38 x^{*}$ & 0.960 & 20.38 & 560 & 570 & 0.98 \\
Полифиламент & $y=-52.29+8.20 x^{*}$ & 0.975 & 8.20 & 590 & 570 & 1.04 \\
& $y=-130.45+23.02 x$ & 0.973 & 23.02 & 290 & 280 & 1.04 \\
& $y=-168.44+29.80 x^{*}$ & 0.980 & 29.80 & 286 & 280 & 1.02 \\
& $y=-85.99+15.20 x^{*}$ & 0.986 & 15.20 & 287 & 280 & 1.03
\end{tabular}

Пр име чан и е. * аппроксимация при использовании двух линейных участков с различными наклонами.

с монофиламентом $(m=12.02)$, что свидетельствует о существенно более узком распределении экспериментальных значений прочности для полифиламентных образцов.

В принципе, при более детальном рассмотрении кривых $\ln \ln \left[1 /\left(1-P_{j}\right)\right]=f(\ln \sigma)$, приведенных на рис. $2, b$, каждая из рассмотренных зависимостей, в особенности кривая для моноволокна, может быть описана при использовании двух аппроксимирующих прямых с различными наклонами: $m=20.38$ и $m=8.20$ для моноволокна и $m=29.80$ и $m=15.20$ для полифиламентного образца. В этом случае, результаты компьютерной 
обработки экспериментальных значений прочности с $R^{2}=0.980$ и 0.986 (по сравнению с $R^{2}=0.973$ при использовании прямой с одним наклоном) и $R^{2}=0.960$ и 0.975 (по сравнению с $R^{2}=0.954$ для прямой с одним наклоном) для поли- и монофиламентных образцов, соответственно, оказываются более корректными. Отметим, что более крутые участки, соответствующие по определению меньшему разбросу данных, характерны для более низких значений $\sigma$ для образцов двух типов.

На рис. 2, $c, d$ приведены гистограммы функции плотности вероятности (probability density function - PDF), полученные при использовании общепринятого подхода (см., например, $[24,25])$, для прочности единичного волокна $(c)$ и полифиламента $(d)$, проанализированные при компьютерной обработке данных, приведенных на рис. 2, $a$, при использовании функции Гаусса. Результаты аппроксимации показаны сплошными линиями. Из полученных результатов обработки следует, что, в целом, гистограммы для образцов двух типов могут быть удовлетворительно описаны с помощью огибающих колоколообразных кривых. Однако теоретическая кривая распределения для полифиламента (см. рис. $2, d$ ) более корректно описывает экспериментальные данные $\left(R^{2}=0.751\right)$, по сравнению с кривой для монофиламента (см. рис. 2, $c, R^{2}=0.563$ ). Следовательно, описание распределения прочности ориентированных волокон ПП в виде полифиламентов является более корректным по сравнению с описанием распределения прочности ориентированных волокон ПП в виде монофиламентов при использовании как функции Вейбулла, так и функции Гаусса. Другими словами, тип образца оказывает заметное влияние на степень однородности распределения прочности. Такое поведение может быть обусловлено следующими причинами.

Как мы отмечали ранее, сам полифиламентный образец (жгут), включающий в себя несколько сотен $(\sim 200)$ тонких моноволокон (филаментов), представляет собой объект, который, априори, наделен статистической природой и является статистически более однородным по сравнению с моноволокном. Интегральная механическая нагрузка, приложенная к жгуту, будет распределяться сравнительно равномерно между его многочисленными филаментами. Поэтому наличие единичных опасных структурных дефектов (поверхностных микротрещин, инициирующих процесс разрушения) в некоторых моноволокнах такого жгута не обязательно будет приводить к разрушению всего образца, поскольку распространение трещины в одном филаменте остановится при встрече с другим филаментом. Эта ситуация коренным образом отличается от деформирования моноволокна, для которого наличие даже единственного опасного дефекта (одной микротрещины) может инициировать процесс разрушения всего образца.

Также отметим, что результаты линейной аппроксимации, полученные при использовании модели Вейбулла $\left(R^{2}>0.95\right)$, представляются более корректными по сравнению с результатами аппроксимации, полученными при использовании колоколообразных кривых Гаусса $\left(R^{2}<0.76\right)$. Полученный результат означает, что, несмотря на более сложный характер колоколообразных зависимостей по сравнению с линейными зависимостями, предполагающий меньшую сходимость данных (уменьшение $R^{2}$ ) в первом случае, механизм разрушения исследованных моно- и полифиламентных волокон ПП в большей степени соответствует квазихрупким материалам, чем пластичным.

При использовании описанных выше подходов проанализируем характер статистических распределений еще одной механической характеристики при разрушении ориентированных полифиламентов ПП - деформации при разрыве $\varepsilon_{b}$. С этой целью два больших массива измеренных значений $\varepsilon_{b}$ (по 50 значений для двух типов образцов), рассмотренных на рис. $3, a$, были перестроены в координатах Вейбулла $\ln \ln \left[1 /\left(1-P_{j}\right)\right]=f\left(\ln \varepsilon_{b}\right)$ (см. рис. 3,b). Затем была проведена их линейная аппроксимация при компьютерной обработке с целью выяснения их соответствия уравнению (7). Результаты проведенного анализа приведены в табл. 2. Видно, что при использовании аппроксимирующей прямой с одним наклоном (стандартная функция распределения Вейбулла) полученная зависимость характеризуется коэффициентами детерминации $R^{2}=0.94$ и 0.97 для моно- и полифиламентных волокон соответственно.

Это означает, что результат аппроксимации представляется корректным для полифиламента, однако для монофиламента целесообразнее использование двух касательных, так как при переходе от единичного наклона к двум наклонам величина $R^{2}$ увеличивается от 0.94 до 0.97. Проведение аналогичной процедуры для анализа кривой для полифиламента также позволяет существенно повысить надежность аппроксимации (увеличение $R^{2}$ от 0.97 до 0.99). Следовательно, в любом случае, при сопоставлении результатов аппроксимации при использовании как двух отрезков прямых с различными наклонами, так и одной прямой с единичным наклоном значения $m$ для полифиламента всегда оказываются в $2-2.5$ раза больше соответствующих значений $m$ для монофиламента.

Таким образом, как и в рассмотренном выше случае для разрывной прочности, распределение деформации при разрыве для образцов полифиламентных волокон ПП является существенно более узким по сравнению с ее распределением для единичных волокон ПП.

Отметим также, что отношение параметра $\varepsilon_{0}$, рассчитанного с помощью уравнения (8), к среднему значению $\varepsilon_{b}, \varepsilon_{\mathrm{av}}$, во всех рассмотренных случаях составляет $\varepsilon_{0} / \varepsilon_{\mathrm{av}} \approx 1$ (см. табл. 2), что также подтверждает корректность применения модели Вейбулла для анализа статистического распределения $\varepsilon_{b}$ высокопрочных монои полифиламентов ПП.

Результаты статистического анализа значений $\varepsilon_{b}$ в рамках модели Гаусса приведены на рис. 3, c, d. Видно, что кривая распределения для полифиламента (см. 

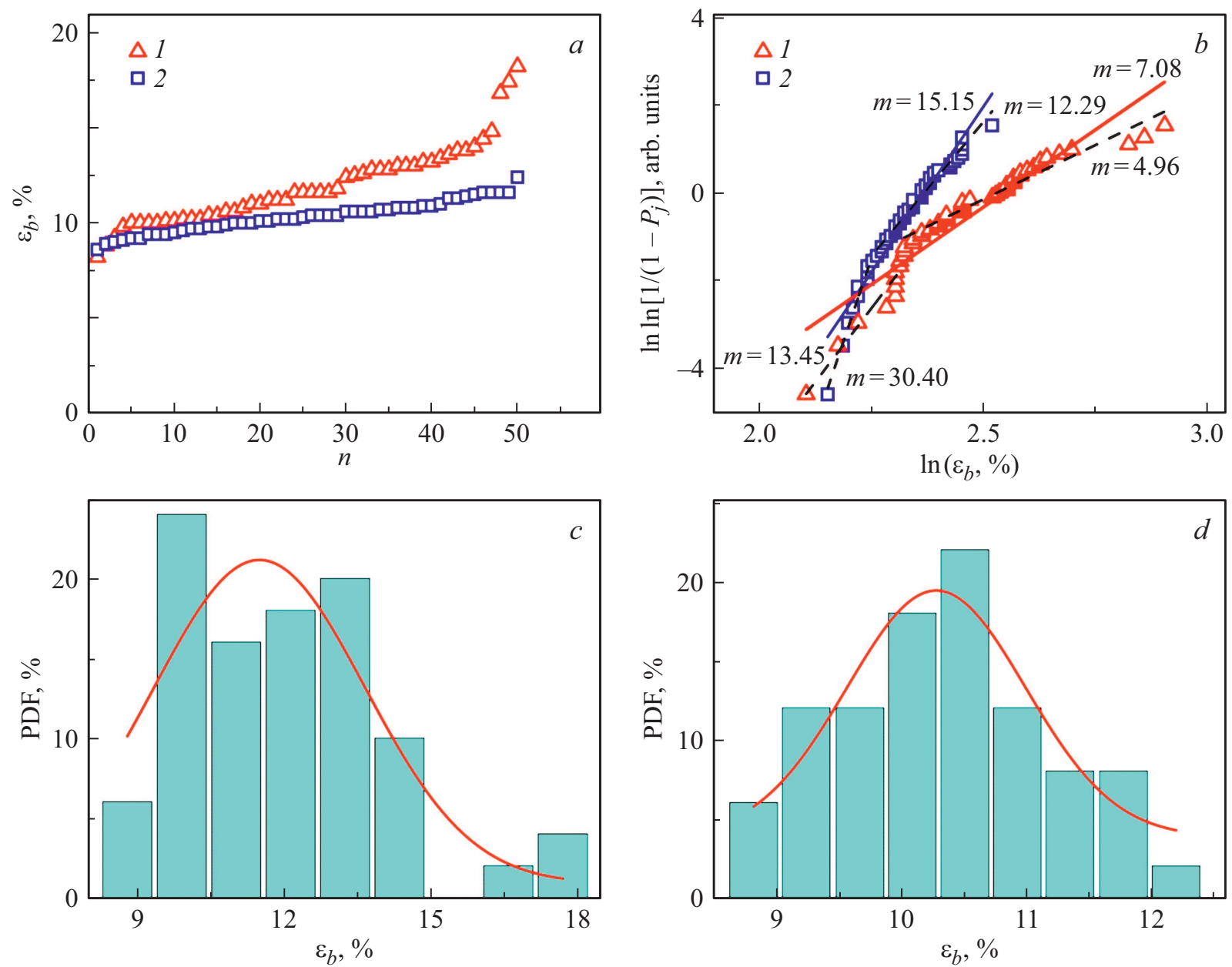

Рис. 3. $a$ - деформация при разрыве $\varepsilon_{b}$ в порядке возрастания ее величины в зависимости от номера образца $n$ для единичных (1) и полифиламентных волокон ориентированного ПП (2); $b$ - графики Вейбулла, построенные на основании данных, представленных в $(a)$ (обозначения те же); сплошными и пунктирными линиями показаны результаты линейной аппроксимации при использовании прямой с одним наклоном и двух прямых с различными наклонами соответственно; $c, d-$ гистограммы функции плотности вероятности (PDF) для деформации при разрыве единичного волокна $(c)$ и полифиламента ПП $(d)$ и их описание при использовании функции Гаусса (сплошные колоколообразные кривые).

Таблица 2. Результаты анализа распределения деформации при разрыве высокопрочных моно- и полифиламентных волокон полипропилена в рамках модели Вейбулла

\begin{tabular}{c|c|c|c|c|c|c}
\hline Тип образца & $y=a+b x$ & $R^{2}$ & $m$ & $\varepsilon_{0} \%$ & $\varepsilon_{\mathrm{av}}, \%$ & $\varepsilon_{0} / \varepsilon_{\mathrm{av}}$ \\
\hline Монофиламент & $y=-18.01+7.08 x$ & 0.937 & 7.08 & 12.7 & 11.7 & 1.09 \\
& $y=-32.89+13.45 x^{*}$ & 0.966 & 13.45 & 11.6 & 11.7 & 0.99 \\
Полифиламент & $y=-12.54+4.96 x^{*}$ & 0.974 & 4.96 & 12.6 & 11.7 & 1.08 \\
& $y=-35.88+15.15 x$ & 0.971 & 15.15 & 10.7 & 10.3 & 1.04 \\
& $y=-69.86+30.40 x^{*}$ & 0.988 & 30.40 & 9.97 & 10.3 & 0.97 \\
& $y=-29.09+12.29 x^{*}$ & 0.988 & 12.29 & 10.7 & 10.3 & 1.00
\end{tabular}

Пр и ме чание. * аппроксимация при использовании двух линейных участков с различными наклонами.

рис. $3, d)$ является более симметричной по сравнению с кривой для монофиламента (см. рис. 3,c). Кроме того, аппроксимация для полифиламента характеризуется более высоким значением $R^{2}=0.766$ по сравнению с монофиламентом $\left(R^{2}=0.593\right)$. Следовательно, опи- сание распределения $\varepsilon_{b}$ в рамках модели Гаусса для полифиламента является более корректным, чем для монофиламента.

Из сопоставления результатов компьютерной обработки значений $\varepsilon_{b}$ при использовании двух различ- 
ных статистических моделей следует, что результаты линейной аппроксимации, полученные при использовании модели Вейбулла $\left(R^{2}>0.93\right)$, представляются более корректными по сравнению с результатами аппроксимации, полученными при использовании колоколообразных кривых Гаусса $\left(R^{2}<0.77\right)$, как и в рассмотренном выше случае для прочности. Они подтверждают высказанное выше предположение о соответствии механизма разрушения исследованных монои полифиламентных волокон ПП механизму разрушения квазихрупких материалов, в котором ключевую роль в разрушении образца играют поверхностные трещины.

Рассмотрим особенности статистических распределений еще одной очень важной механической характеристики при практическом применении высокопрочных ориентированных волокон ПП - их модуля упругости $E$. С этой целью два массива значений $E$ для моно- и полифиламентов ПП, представленные на рис. 4, $a$, были проанализированы в соответствии с моделями Вейбулла (см. рис. 4, $b$ и табл. 3) и Гаусса (см. рис. 4,c,d). Как следует из результатов компьютерной обработки графиков Вейбулла, приведенных на рис. 4, $b$ и в табл. 3, аппроксимация прямой с одним наклоном может считаться удовлетворительной лишь для полифиламентов ПП $\left(m=21.54, R^{2}=0.939\right)$. Для монофиламентов ПП она является неудовлетворительной $(m=11.20$, $\left.R^{2}=0.866\right)$, что требует аппроксимации с использованием двух касательных прямых к анализируемой кривой. Данная процедура дает значения $m=125.75$ и $m=7.71$ для областей с более низкими и более высокими значениями $E$, соответственно.

Отметим, что очень высокое значение $m \approx 126$, рассчитанное для данной статистической характеристики в области более низких значений $E<8 \mathrm{GPa}$, не представляется нам нереалистичным, так как оно соизмеримо со значениями $m=74-76$ и $m=98$, полученными для сверхпрочных $(\sigma=4-6 \mathrm{GPa})$ волокон органической (СВМПЭ [3,26]) и неорганической природы [7-13], соответственно. Факт существенного, более чем на один десятичный порядок, превышения значения $m=126$ значения $m \approx 8$, рассчитанного для области более высоких значений $E>8 \mathrm{GPa}$, свидетельствует о резком уменьшении разброса данных для монофиламентов ПП в области более низких значений $E$. Можно думать, что некоторая часть испытанных монофиламентов ПП $(\sim 25 \%)$ в силу сравнительно большого для волокон диаметра $(170 \mu \mathrm{m})$ подвергалась в процессе производства менее однородной вытяжке по сечению образца. Это приводило к достижению хотя и более низких, но характеризующихся лучшей воспроизводимостью значений $E$.

Если проанализировать зависимость $\ln \ln \left[1 /\left(1-P_{j}\right)\right]$ $-\ln E$ на рис. 4, $b$ для полифиламентов путем аппроксимации при использовании двух касательных, то значение $m=29.06$, полученное при меньших значениях $E$, будет также больше, чем значение $m=17.98$ для больших значений $E$, как и в случае монофиламента. Однако отношение этих двух значений $m$ оказывается существенно меньше $(\sim 29 / 18<2)$ по сравнению с соответствующим отношением значений $m$ для монофиламентов $(\sim 126 / 8>10)$. Это означает, что разброс значений $E$ для полифиламентов является более однородным по сравнению с монофиламентами.

Отметим также близость средних измеренных значений $E, E_{\mathrm{av}}$, и теоретических значений $E_{0}$, рассчитанных с помощью уравнения (10), для всех выше рассмотренных случаев. Наблюдаемое соблюдение условия $E_{0} \approx E_{\mathrm{av}}$ (см. табл. 3) подтверждает корректность проведенного анализа статистических распределений $E$ в рамках модели Вейбулла.

В заключение рассмотрим гистограммы PDF в зависимости от величины $E$ (см. рис. 4, $c, d$ ) для выяснения их соответствия распределению Гаусса. Как следует из данных рис. 4,c, при компьютерной обработке экспериментальных данных получить огибающую колоколообразную кривую для единичного волокна не удается. В то же время, для полифиламента (см. рис. 4,d) такая кривая, характерная для нормального распределения (или распределения Гаусса), наблюдается. Выявленное различие может быть обусловлено следующими причинами. Нам представляется, что в случае полифиламентов, состоящих из нескольких сотен очень тонких $(\sim 1 \mu \mathrm{m})$ мононитей (монофиламентов), приложенная интегральная механическая нагрузка при растяжении усредняется между большим количеством монофиламентов, что не позволяет обнаружить резкую границу перехода изменения $E$, выявленную для единичного волокна. Такое поведение также может быть объяснено более совершенной ориентационной вытяжкой при получении монофиламентов (ПП) по сравнению с технологией производства полифиламентов ПП, которая подтверждается более высокими (в 2 раза) значениями $E$ и разрывной прочности для монофиламентов по сравнению со значениями $E$ и $\sigma$ для полифиламентов (см. рис. 4, $a$ и рис. $2, a)$.

В сводной табл. 4 обобщены результаты статистического анализа кривых распределения $\sigma, \varepsilon_{b}$ и $E$ для высокопрочных моно- и полифиламентных волокон ПП, полученные при линейной аппроксимации графиков Вейбулла при использовании одного наклона. Интересно отметить, что для всех трех исследованных механических характеристик значение $m$ для полифиламентов всегда в $\sim 2$ раза превышает соответствующее значение $m$ для монофиламентов. Другими словами, установленная разница между значениями $m$ для образцов двух типов является константой, причем как для модуля упругости $(E)$, так и для разрывных механических характеристик $(\sigma$ и $\left.\varepsilon_{b}\right)$. Данное обстоятельство представляется весьма неожиданным, так как эти два различных типа механических характеристик контролируются различными молекулярно-структурными механизмами. Как известно [3,7-12], в случае разрывных характеристик крити- 

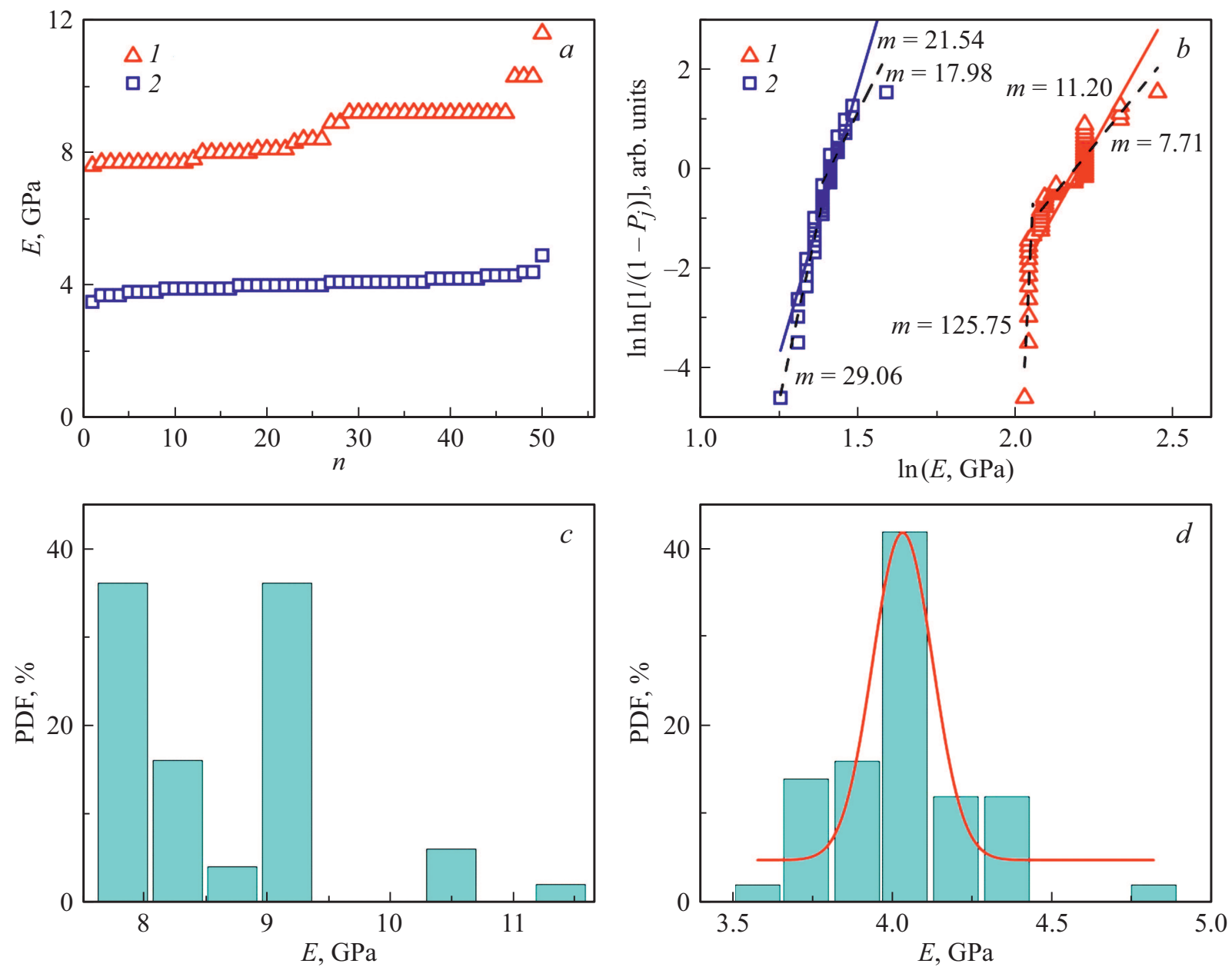

Рис. 4. $a$ - модуль упругости $E$ в порядке возрастания его значения в зависимости от номера образца $n$ для единичных волокон $(1)$ и полифиламентов ориентированного ПП $(2) ; b$ - графики Вейбулла, построенные из данных, представленных в $(a)$; сплошными и пунктирными линиями показаны результаты линейной аппроксимации при использовании прямой с одним наклоном и двух прямых с разными наклонами, соответственно; $c, d-$ гистограммы функции плотности вероятности (РDF) для модуля упругости единичного волокна $(c)$ и полифиламента ПП $(d)$; сплошная колоколообразная кривая на $(d)$ - результат компьютерной обработки при использовании функции Гаусса.

Таблица 3. Результаты анализа распределения модуля упругости высокопрочных моно- и полифиламентов полипропилена в рамках модели Вейбулла

\begin{tabular}{c|l|c|c|c|c|c}
\hline Тип образца & \multicolumn{1}{|c|}{$y=a+b x$} & $R^{2}$ & $m$ & $E_{0}$, ГПа & $E_{c p}$, ГПа & $E_{0} / E_{c p}$ \\
\hline Монофиламент & $y=-24.67+11.20 x$ & 0,866 & 11.20 & 9.06 & 8.64 & 1.05 \\
& $y=-259.02+125.75 x^{*}$ & 0.723 & 125.75 & 7.85 & 8.64 & 0.91 \\
& $y=-16.88+7.71 x^{*}$ & 0.927 & 7.71 & 8.94 & 8.64 & 1.03 \\
Полифиламент & $y=-30.64+21.54 x$ & 0.939 & 21.54 & 4.15 & 4.00 & 1.04 \\
& $y=-40.95+29.06 x^{*}$ & 0.969 & 29.80 & 4.10 & 4.00 & 1.02 \\
& $y=-25.38+17.98 x^{*}$ & 0.964 & 17.98 & 4.11 & 4.00 & 1.03
\end{tabular}

Пр име чан и е. * аппроксимация при использовании двух линейных участков с различными наклонами.

ческую роль играют поверхностные трещины, тогда как в случае определения $E$, на начальных стадиях деформирования, роль трещин представляется пренебрежимо малой, а основную, ключевую роль отводят жесткости индивидуальной цепи.
Более высокие значения $m$ для полифиламентов, свидетельствующие о меньшем разбросе данных, могут быть связаны с уже отмечавшейся нами статистической природой полифиламентного образца, обеспечивающей более равномерное по сравнению с моноволокном рас- 
Таблица 4. Значения статистического модуля Вейбулла $m$, рассчитанные из кривых распределения $\sigma, \varepsilon_{b}$ и $E$ для высокопрочных моно- и полифиламентных волокон полипропилена при линейной аппроксимации с использованием одного наклона

\begin{tabular}{l|r|r|r}
\hline \multirow{2}{*}{\multicolumn{1}{c|}{ Тип образца }} & \multicolumn{3}{|c}{ Характеристика } \\
\cline { 2 - 4 } & \multicolumn{1}{c|}{$\sigma$} & \multicolumn{1}{c|}{$\varepsilon_{b}$} & \multicolumn{1}{c}{$E$} \\
\hline Монофиламентное волокно & 12.02 & 7.08 & 11.20 \\
Полифиламентное волокно (жгут) & 23.02 & 15.15 & 21.54
\end{tabular}

пределение приложенной механической нагрузки между многочисленными филаментами.

Что касается результатов аппроксимации кривых распределения $\sigma, \varepsilon_{b}$ и $E$ в рамках модели Гаусса, то для полифиламентов ПП типичные для данного типа распределения колоколообразные кривые были получены для всех этих трех исследованных механических характеристик (см. рис. $2, d, 3, d$ и $4, d$ ). В то же время, для монофиламентов ПП такие кривые могут быть получены только для прочности и деформации при разрыве (см. рис. $2, c$ и $3, c$ ), причем их форма является существенно более асимметричной по сравнению с полифиламентами. Другими словами, в целом, результаты компьютерной обработки для монофиламентов представляются менее корректными, чем для полифиламентов.

Таким образом, статистические распределения всех трех исследованных механических характеристик: прочности, деформации при разрыве и модуля упругости, для полифиламентов ПП могут быть корректно описаны как в рамках модели Вейбулла, так и в рамках модели Гаусса при анализе одних и тех же экспериментальных результатов. Нам представляется, что в данном случае проявляется ,дуализм“ статистики $\sigma, \varepsilon_{b}$ и $E$, наблюдавшийся нами ранее для прочности полифиламентных волокон ПА-6 [28] - полимера с другим типом конформации цепи: плоским транс-зигзагом.

Выявленное в настоящей работе проявление статистического дуализма распределения упругих и деформационно-прочностных механических характеристик для материалов на основе ПП может быть обусловлено следующими причинами. С одной стороны, хотя значения $\varepsilon_{b}=8-17 \%$ для исследованных пластичных полипропиленовых материалов превосходят значения $\varepsilon_{b}<5 \%$ для квазихрупких материалов, для которых модель Вейбулла оказалась наиболее эффективной, оба интервала деформаций $\varepsilon_{b}$ являются величинами одного порядка. Поэтому применимость модели Вейбулла оказывается возможной также и в интервале $\varepsilon_{b}$ от 8 до $17 \%$. С другой стороны, оказалось, что статистика Гаусса в случае полифиламентных образцов также применима, поскольку этот материал является статистически более однородным (одновременно деформируется несколько сотен единичных волокон). Такая система соответствует нормальному распределению процесса разрушения по сечению образца, независимо от его механизма. Кроме того, полифиламентные волокна ПП характеризуются сравнительно невысокими значениями модуля упругости $E=3-5 \mathrm{GPa}$ и большими значениями деформации при разрыве $(8-17 \%)$, т.е. они обладают определенным ресурсом пластичности. Данное обстоятельство является также благоприятным фактором для возможности корректного описания статистического распределения механических характеристик в рамках модели Гаусса.

Значения $m=7-23$, определенные в настоящей работе для высокопрочных моно- и полифиламентных волокон ПП (см. табл. 4), в особенности для полифиламентов $(m=15-23)$, являются более высокими по сравнению со значениями $m$ из литературных источников для сверхпрочных ультраориентированных (вытянутых в 120 раз) образцов СВМПЭ $(m=7-10)$ [26,27,30], кварцевых $(m=12)$ [9], керамических $(m=8-9)$ [7], стеклянных и углеродных волокон $(m=3-11)[8,13]$, а также углеродных нанотрубок $(m=3-7)$ [11]. Это свидетельствует о меньшем разбросе экспериментальных данных в случае материалов на основе ПП, что может быть объяснено более выраженной пластичностью исследованных в работе волокон ПП (большей деформацией при разрыве $\left.\varepsilon_{\mathrm{av}}=10-11 \%\right)$ по сравнению с квазихрупкими сверхпрочными материалами $\left(\varepsilon_{\mathrm{av}}<5 \%\right)$. Этот аргумент коррелирует с наблюдением более высоких значений $m=43-45$ для прочности достаточно пластичного высокопрочного материала - полиамида-6 $\left(\varepsilon_{b}=16 \%\right)[28,29]$. Кроме того, прочность пластичных волокон ПП $\left(\varepsilon_{\mathrm{av}}=0.3-0.6 \mathrm{GPa}\right)$ является существенно, на один десятичный порядок, более низкой по сравнению с прочностью хрупких сверхпрочных материалов $\left(\varepsilon_{\mathrm{av}}=2-6 \mathrm{GPa}\right)$, что также может приводить к увеличению разброса данных (уменьшению значений $m$ ) для последних.

Следует также отметить, что значения модуля Вейбулла $m=7-23$ для материалов на основе ПП (спиралевидная конформация цепи) являются промежуточными между значениями $m=7-10$ и $m=43-45$ [3,26-30], рассчитанными нами для материалов на основе СВМПЭ и ПА-6, соответственно, имеющих конформацию плоского транс-зигзага. Следовательно, влияние типа конформации цепи на величину разброса механических характеристик ориентированных волокон полимеров является неоднозначным. Его выяснение требует дальнейшего проведения дополнительных исследований с привлечением полимерных материалов с другими типами химической структуры цепи.

\section{5. Заключение}

На примере высокопрочных полимерных материалов - полифиламентных волокон ПП со спиралевидной конформацией цепи - впервые показано, что распределения упругих $(E)$ и деформационно-прочностных механических характеристик $\left(\sigma\right.$ и $\left.\varepsilon_{b}\right)$ могут быть корректно 
описаны при использовании двух статистических подходов, Вейбулла и Гаусса, основывающихся на различных концепциях описания вероятности механического разрушения материалов. Полученные данные подтверждают проявление статистического дуализма распределения механических свойств, обнаруженного нами ранее [2630] для высокопрочных материалов на базе других полимеров - СВМПЭ и ПА-6 с конформацией цепи типа плоский транс-зигзаг.

На основе большого массива статистических данных (50 измерений идентичных образцов для материала каждого типа) определены параметры распределения Вейбулла для ориентированных моно- и полифиламентных волокон ПП. Установлено, что значения статистического модуля Вейбулла $m$ - характеристики разброса данных - для каждого из трех исследованных механических свойств полифиламентных волокон в 2 раза превышают соответствующие значения $m$ для единичных волокон. Это означает, что разброс данных при механических испытаниях полифиламентных волокон является существенно меньшим, чем для моноволокон. Данный результат связывается с более выраженной статистической однородностью полифиламентных волокон, состоящих из нескольких сотен тонких единичных волокон. Полученные в настоящей работе данные подтверждают существенное влияние типа образца на специфику разброса экспериментальных данных, обнаруженную ранее для сверхпрочных материалов на основе СВМПЭ [30]. Показано, что влияние конформации цепи на величину разброса механических характеристик является неоднозначным и требует дальнейшего выяснения.

\section{Конфликт интересов}

Авторы заявляют, что у них нет конфликта интересов.

\section{Список литературы}

[1] V.A. Marikhin, L.P. Myasnikova. Structural basis of highstrength high-modulus polymers. In: Oriented Polymer Materials / Ed. S. Fakirov. Huthig \& Wepf Verlag-Zug, Heidelberg (1996). P. 38-98.

[2] V. Marikhin, L. Myasnikova, Y. Boiko, E. Ivan'kova, E. Radovanova, P. Yakushev. Role of Reactor Powder Morphology in Producing High-strength High-modulus UHMWPE Fibres. In: Reactor Powder Morphology / Ed. L. Myasnikova, P. Lemstra. Nova Publishers, Hauppauge, N.Y, Ch. 10 (2011). P. 235-294.

[3] Yu.M. Boiko, V.A. Marikhin, L.P. Myasnikova, O.A. Moskalyuk, E.I. Radovanova. J. Mater. Sci. 52, 3, 1727 (2017).

[4] Yu.M. Boiko, V.V. Kovriga. Intern. J. Polym. Mater. 22, 1-4, 209 (1993).

[5] R. Marissen, D. Wienke, R. Homminga, R. Bosman, K.M. Veka, A. Huguet. Mater. Sci. Appl. 7, 5, 238 (2016).

[6] Ю.М. Бойко, В.А. Марихин, О.А. Москалюк, Л.П. Мясникова. ФТТ 61, 1, 182 (2019).

[7] D.M. Wilson. J. Mater. Sci. 32, 10, 2535 (1997). doi: 10.1023/A:1018538030985.
[8] F. Tanaka, T. Okabe, H. Okuda, I.A. Kinloch, R.J. Young. Composites A, 57, 2, 88 (2014).

[9] Л.Г. Байкова, Т.И. Песина, М.Ф. Киреенко, Л.В. Тихонова, C.R. Kurkjian. ЖТФ 85, 6, 83 (2015).

[10] K.-H. Nitta, C.-Y. Li. Physica A 490, 1076 (2018). doi: 10.1016/j.physa.2017.08.113.

[11] A.H. Barber, R. Andrews, L.S. Shaudler, H.D. Wagner. Appl. Phys. Lett. 87, 203106 (2005).

[12] G. Sun, J.H.L. Pang, J. Zhou, Y. Zhang, Z. Zhan, L. Zheng. Appl. Phys. Lett. 101, 131905 (2012).

[13] M.R. Gurvich, A.T. Dibenedetto, A. Pegoretti. J. Mater. Sci. 32, 14, 3711 (1997).

[14] M. Aguiari, M. Palombo, C.M. Rizzo. Welding World 65, 289 (2021).

[15] C. Maraveas, Z.C. Fasoulakis, K.D. Tsavdaridis. Fire Sci. Rev. 6, 3, 1 (2017).

[16] Y. Huang, X. Jin, G. Cai. J. Mater. Res. 32, 20, 3894 (2017).

[17] Ю.М. Бойко, И.Г. Кузнецова, В.В. Коврига, А.Я. Гольдман, А.М. Тарасов, В.А. Артемьев, Г.Д. Мясников. Мех. композ. матер. 23, 2, 202 (1987).

[18] Ю.М. Бойко, В.В. Коврига, А.Я. Гольдман. Высокомолекуляр. соединения А 33, 9, 1972 (1991).

[19] Yu.M. Boiko, W. Brostow, A.Ya. Gol'dman, A.C. Ramamurthy. Polymer 36, 7, 1383 (1995).

[20] L. Laiarinandrasana, Y. Nziakou, J.L. Halary. J. Polym. Sci. B 50, 1740 (2012).

[21] Ю.М. Бойко, М.Я. Шерман. Высокомолекуляр. соединения A 40, 2, 279 (1998).

[22] Ю.М. Бойко, И.Г. Кузнецова, С.А. Унежева, В.В. Коврига, Л.А. Ганн. Мех. композ. матер. 29, 1, 3 (1993).

[23] W. Weibull. J. Appl. Mech. 18, 9, 293 (1951).

[24] Е.И. Куликов. Прикладной статистический анализ (2008). $464 \mathrm{c}$.

[25] J.K. Patel, C.B. Read. Handbook of the Normal Distribution. 2nd ed. Statistics: Handbooks and Monographs. 150, N.Y. Marcel Dekker, Inc (1996). 456 p. ISBN 0-8247-9342-0.

[26] Ю.М. Бойко, В.А. Марихин, О.А. Москалюк, Л.П. Мясникова, Е.И. Радованова. ФТТ 58, 10, 2065 (2016).

[27] Yu.M. Boiko, V.A. Marikhin, L.P. Myasnikova, E.I. Radovanova. Colloid Polym. Sci. 296, 10, 1651 (2018).

[28] Ю.М. Бойко, В.А. Марихин, О.А. Москалюк, Л.П. Мясникова, Е.С. Цобкалло. Письма ЖТФ 45, 8, 37 (2019).

[29] Ю.М. Бойко, В.А. Марихин, О.А. Москалюк, Л.П. Мясникова, Е.С. Цобкалло. Письма ЖТФ 45, 14, 20 (2019).

[30] Ю.М. Бойко, В.А. Марихин, О.А. Москалюк, Л.П. Мясникова. ФТТ 62, 4, 590 (2020).

[31] П. Флори. Статистическая механика цепных молекул. Мир, М. (1971). 440 c.

[32] J.D. Sullivan, P.H. Lauzon. J. Mater. Sci. Lett. 5, 1245 (1986).

Редактор К.В. Емцев 